\title{
A STURMIAN THEOREM FOR FIRST ORDER PARTIAL DIFFERENTIAL EQUATIONS
}

\author{
BY \\ PUI-KEI WONG
}

\begin{abstract}
A pair of first order partial differential equations is considered. The system is transformed into a single nonlinear scalar equation of the Riccati type from which some Wirtinger type integral inequalities for functions of several variables are derived. A comparison theorem for two such pairs of first order equations is then proved using the Wirtinger inequalities.
\end{abstract}

Let $G$ be a bounded domain of $d$-dimensional Euclidean space $R^{d}$ with boundary $\partial G$. We assume that $G$ can be approximated from within by a sequence $\left\{G_{n}\right\}$ of bounded domains each having a smooth boundary $\partial G_{n}$ such that $G_{n} \subset \bar{G}_{n} \subset G_{n+1}$ $\subset \bar{G}_{n+1} \subset G$ and $\bigcup G_{n}=G$. Variable points of $R^{d}$ will be denoted by $x=\left(x_{1}, \ldots, x_{d}\right)$ and differentiation with respect to $x_{i}$ is denoted by $D_{i}$. Real scalar quantities will be represented by lower case Latin letters $u, v, w$, etc.; vectors in $R^{d}$ will be denoted by small Greek letters $\alpha, \beta, \gamma$, etc.; and matrices will be denoted by capital Latin letters $A, B, C$, etc. The inner product between two vectors $\alpha$ and $\beta$ will be written as $\alpha \cdot \beta$ while the length of $\alpha$ is denoted by $\|\alpha\|$. We consider the pair of first order partial differential equations

$$
\nabla u=u \alpha(x)+B(x) \zeta, \quad \nabla \cdot \zeta=-p(x) u+\beta(x) \cdot \zeta
$$

where $p, \alpha, \beta$, and $B$ are continuous in $G$ and $B$ is symmetric and positive definite there. The following is immediate.

Lemma 1. Let $(u, \zeta)$ be a solution of (1) such that $u(x) \neq 0$ in $G$. Define

$$
\varphi(x)=u^{-1}(x) \zeta(x) .
$$

\section{Then $\varphi$ satisfies the generalized Riccati equation}

$$
\nabla \cdot \varphi+B \varphi \cdot \varphi+(\alpha-\beta) \cdot \varphi+p=0 .
$$

Since $B$ is symmetric and positive definite, $B^{-1}$ exists and is symmetric and positive definite in $G$. We introduce the functionals $M\left(w: G_{n}\right)$ and $Q\left(w: G_{n}\right)$ defined respectively by

$$
M\left(w: G_{n}\right)=\int_{G_{n}} B^{-1}(\nabla w-w B \varphi) \cdot(\nabla w-w B \varphi) d x
$$

Received by the editors December 30, 1970.

AMS 1970 subject classifications. Primary 35F15; Secondary 26A84.

Key words and phrases. Comparison theorems, first order equations, generalized Riccati equation, Wirtinger inequalities. 
and

$$
Q\left(w: G_{n}\right)=\int_{G_{n}}\left(B^{-1} \nabla w \cdot \nabla w-p w^{2}\right) d x,
$$

where $w \in C^{1}(G)$ and $\varphi$ is any $C^{1}$-solution of (3).

LEMMA 2. Let $\varphi$ be any $C^{1}$-solution of (3) in $G$ and let $G_{n} \in\left\{G_{n}\right\}$. Then for every $w \in C^{1}(G)$,

$$
\int_{\partial G_{n}} w^{2}(\varphi \cdot \eta) d S \leqq Q\left(w: G_{n}\right)+\int_{G_{n}} w^{2}(\beta-\alpha) \cdot \varphi d x,
$$

where $\eta=\left(\eta_{1}, \ldots, \eta_{d}\right)$ denotes the outward pointing unit normal on $\partial G_{n}$. Moreover, equality holds in (6) if, and only if, $w$ satisfies

$$
\nabla w \equiv w(B \varphi) .
$$

Proof. Since $w \in C^{1}(G)$ and $\bar{G}_{n} \subset G$, the integral (4) is well defined. Expanding (4) one gets

$$
M\left(w: G_{n}\right)=\int_{G_{n}}\left\{B^{-1} \nabla w \cdot \nabla w+w^{2}(B \varphi \cdot \varphi)-2 w \nabla w \cdot \varphi\right\} d x .
$$

Since $\nabla \cdot\left(w^{2} \varphi\right)=2 w \nabla w \cdot \varphi+w^{2} \nabla \cdot \varphi$, the last integral in (8) above can be integrated by parts once by means of Green's formula:

$$
-2 \int_{G_{n}}(w \nabla w \cdot \varphi) d x=\int_{G_{n}} w^{2} \nabla \cdot \varphi d x-\int_{\partial G_{n}} w^{2}(\varphi \cdot \eta) d S .
$$

Putting this into (8) and using the fact that $\varphi$ satisfies (3), we arrive at

$$
0 \leqq M\left(w: G_{n}\right)=Q\left(w: G_{n}\right)+\int_{G_{n}} w^{2}(\beta-\alpha) \cdot \varphi d x-\int_{\partial G_{n}} w^{2}(\varphi \cdot \eta) d S
$$

from which (6) follows. It is clear that equality will hold if, and only if, $M\left(w: G_{n}\right)=0$ for every $n$, i.e., $\nabla w \equiv w B \varphi$.

Lemma 2 can be stated as a Wirtinger type inequality for certain classes of functions of several variables. To see this we suppose $\varphi$ is a given solution of (3). Denote by $\Omega$ all those functions $w \in C^{1}(G)$ for which $M(w)=\lim _{n} M\left(w: G_{n}\right)$, $Q(w)=\lim _{n} Q\left(w: G_{n}\right)$, and

$$
\int_{\partial G} w^{2}(\varphi \cdot \eta) d S=\lim _{n} \int_{\partial G_{n}} w^{2}(\varphi \cdot \eta) d S
$$

all exist.

THEOREM 1. Let $\varphi$ be a solution of (3) such that $(\alpha-\beta) \cdot \varphi \geqq 0$ in $G$. Then for every $w \in \Omega$,

$$
\int_{\partial G} w^{2}(\varphi \cdot \eta) d S \leqq Q(w)
$$


Moreover, if $(\alpha-\beta) \cdot \varphi \equiv 0$ in $G$, then equality will hold in (9) if, and only if, $w$ is a solution of (7).

Combining this with Lemma 1 we have

COROLlaRY 1.1. Let $(u, \zeta)$ be a solution of (1) such that $u(x) \neq 0$ and $u^{-1}(\alpha-\beta) \cdot \zeta$ $\geqq 0$ in $G$. Then for every $w \in \Omega$,

$$
\int_{\partial G} w^{2} u^{-1}(\zeta \cdot \eta) d S \leqq Q(w) .
$$

Moreover, if $(\alpha-\beta) \cdot \zeta \equiv 0$ in $G$, then equality holds in (10) if, and only if, $w$ is a solution of

$$
\nabla\left(u^{-1} w\right)=\left(u^{-1} w\right) \alpha .
$$

Corollary 1.2. Suppose in addition $\alpha \in C^{1}(G)$ such that $D_{i} a_{j} \equiv D_{j} a_{i}$, where $\alpha=\left(a_{1}, \ldots, a_{d}\right)$. Let $(u, \zeta)$ be a solution of $(1)$ such that $u(x) \neq 0$ and $(\alpha-\beta) \cdot \zeta \equiv 0$ in $G$. Then, for every $w \in \Omega$, inequality (10) is valid. Moreover, equality holds if, and only if, $w \equiv u \exp f$ for some $f \in C^{1}(G)$.

We need only verify the last statement of the corollary. To do this we set $u^{-1} w=f$. Then equation (11) becomes

$$
\nabla f=\alpha .
$$

According to the theorem of Frobenius [4], when $\alpha \in C^{1}(G)$, a necessary and sufficient condition for (12) to be solvable is that $D_{i} a_{j} \equiv D_{j} a_{i}$. This proves the assertion.

For the case where $\alpha=\beta \equiv 0$, (1) has the form

$$
\nabla u=B \zeta, \quad \nabla \cdot \zeta=-p u .
$$

In this case equality will hold in (10) if, and only if, $w \equiv k u$, where $k$ is a constant.

We remark that the Wirtinger inequality (9) is valid even though the coefficient functions in (3) may have singularities on all or parts of the boundary $\partial G$. The family $\Omega$ of admissible functions must of course be so chosen that the integrals appearing in (6) have finite limits. In the one dimensional case such integral inequalities are well known, cf., [1] and [3, Theorem 253]. In the example below we shall give one such inequality for a plane rectangular domain.

In this example $J_{p}(t)$ will denote as usual the Bessel function of the first kind of order $p$. Let $H=\left\{\left(x_{1}, x_{2}\right) \in R^{2}: 0<x_{1}<t^{*}, 0<x_{2}<2 \pi / \sqrt{ } 3\right\}$, where $t^{*}$ denotes the first zero of $J_{3 / 4}^{\prime}(t)$ to the right of the origin $t=0$. The boundary $\partial H$ consists of four edges $\gamma_{i}, i=1, \ldots, 4$, where

$$
\begin{aligned}
& \gamma_{1}=\left\{\left(x_{1}, x_{2}\right) \in R^{2}: 0 \leqq x_{1}<t^{*}, x_{2}=2 \pi / \sqrt{ } 3\right\} \\
& \gamma_{2}=\left\{\left(x_{1}, x_{2}\right) \in R^{2}: x_{1}=0,0 \leqq x_{2}<2 \pi / \sqrt{ } 3\right\} \\
& \gamma_{3}=\left\{\left(x_{1}, x_{2}\right) \in R^{2}: 0<x_{1} \leqq t^{*}, x_{2}=0\right\}
\end{aligned}
$$


and

$$
\gamma_{4}=\left\{\left(x_{1}, x_{2}\right) \in R^{2}: x_{1}=t^{*}, 0<x_{2} \leqq 2 \pi / \sqrt{ } 3\right\} .
$$

We consider the linear second order equation

$$
\Delta_{2} u+\left(x_{1}\right)^{-1} D_{1} u+\left(x_{1}\right)^{-2} u=0
$$

subject to the boundary conditions

$$
u=0 \quad \text { on } \gamma_{1} \cup \gamma_{2} \cup \gamma_{3}, \quad D_{1} u=0 \text { on } \gamma_{4} .
$$

Equation (13) can be put into the form of (1) by setting $\nabla u=\zeta, \alpha \equiv 0, \beta=\left(-1 / x_{1}, 0\right)$, $p\left(x_{1}, x_{2}\right)=1 / x_{1}^{2}$, and $B=I_{2}$, the $2 \times 2$ identity matrix. Thus the coefficients $p$ and $\beta$ are singular on the left edge $\gamma_{2}$. It is a simple matter to verify that

$$
u\left(x_{1}, x_{2}\right)=J_{3 / 4}\left(x_{1}\right) \sin \left(\sqrt{ } 3 x_{2} / 2\right)
$$

is a solution for (13) and (14). Moreover, $u(x)>0$ and $(\alpha-\beta) \cdot \varphi=-\beta \cdot\left(u^{-1} \nabla u\right)$ $=D_{1} u / u>0$ in $H$. Finally, $u$ has a simple zero on $\gamma_{1} \cup \gamma_{3}$, a zero of order $3 / 4$ on $\gamma_{1}$, and $D_{1} u=\nabla u \cdot \eta=0$ on $\gamma_{4}$. If we take as admissible class $\Omega_{H}$ all those functions $w \in C^{1}(H)$ for which $w$ has a zero of order $r>1 / 2$ on $\gamma_{1} \cup \gamma_{2} \cup \gamma_{3}$, then we have the following special example of Theorem 1.

Corollary 1.3. Let $H$ and $\Omega_{H}$ be as defined above. Then for every $w \in \Omega_{H}$

$$
\iint_{H}\|\nabla w\|^{2} d x_{1} d x_{2}>\iint_{H}\left(x_{1}\right)^{-2} w^{2} d x_{1} d x_{2}
$$

unless $w \equiv 0$.

As an application of the Wirtinger type inequality (10) we shall prove a comparison theorem between two first order systems of type (1). To simplify the formulation of such a result we shall let $\Gamma_{0}$ be an arc of $\partial G$ containing a subarc $\gamma_{0}$ on which the coefficient functions may have singularities. Note that the possibility of $\gamma_{0}=\Gamma_{0}=\partial G$ is not excluded. Denote by $\Gamma^{*}=\partial G \mid \Gamma_{0}$. The equations to be compared are

$$
\begin{aligned}
& \nabla u=u \alpha_{1}+B_{1} \zeta, \quad x \in G ; \quad u=0, \quad x \in \Gamma_{0}, \\
& \nabla \cdot \zeta=-p_{1} u+\alpha_{1} \cdot \zeta, \quad x \in G ; \quad \zeta \cdot \eta=g_{1}(x) u, \quad x \in \Gamma^{*} \text {, }
\end{aligned}
$$

and

$$
\begin{array}{rlrlrl}
\nabla w & =w \alpha_{2}+B_{2} \xi, & x \in G ; & w & =0, & x \in \Gamma_{0}, \\
\nabla \cdot \xi & =-p_{2} w+\alpha_{2} \cdot \xi, & x \in G ; & \xi \cdot \eta=g_{2}(x) w, & x \in \Gamma^{*} .
\end{array}
$$

We make the following assumptions:

H1. $B_{1}$ and $B_{2}$ are symmetric positive definite matrices of class $C(\bar{G})$.

H2. $p_{i}, \alpha_{i} \in C(G), i=1,2$, and they can all be extended continuously to $\left(G \cup \Gamma^{*}\right)$.

H3. If $\bar{x} \in \gamma_{0}$ and $\left\{y_{n}\right\}$ is a sequence of points in $G$ such that $y_{n} \in G_{n}$ and $\lim _{n}\left\|y_{n}-\bar{x}\right\|=0$, then the functions $p_{i}$ are of order $O\left(\left\|y_{n}-\bar{x}\right\|^{-2}\right)$ and $\alpha_{i}$ are of order $O\left(\left\|y_{n}-\bar{x}\right\|^{-1}\right)$. 
TheOREM 2. Suppose $\mathrm{H} 1, \mathrm{H} 2$ and $\mathrm{H} 3$. Let $(w, \xi)$ be a solution of (17) such that $w \not 0$ in $G$ and that $w$ has a zero of order $r>1 / 2$ on $\Gamma_{0}$. If $(u, \zeta)$ is a solution of (16) and if

(18) $\int_{G}\left\{\nabla w \cdot\left(B_{1}^{-1}-B_{2}^{-1}\right) \nabla w+\left(p_{2}+\alpha_{2} \cdot B_{2}^{-1} \alpha_{2}-p_{1}\right) w^{2}\right\} d x \leqq \int_{\Gamma^{*}} w^{2}\left(g_{1}-g_{2}\right) d S$,

then $u$ must have a zero in $G$ unless $u$ and $w$ are related by (11).

Proof. We first note that under the given assumptions it is not difficult to verify that all the integrals appearing in (10) and (18) exist. Suppose the contrary conclusion and let $(u, \zeta)$ be a solution of (16) such that $u(x) \neq 0$ in $G$. If we multiply the second equation in (17) by $w$ and integrate by parts once, we have

$$
\begin{aligned}
\int_{G}\left(w \alpha_{2} \cdot \xi-p_{2} w^{2}\right) d x & =\int_{G} w \nabla \cdot \xi d x \\
& =\int_{\partial G} w(\xi \cdot \eta) d S-\int_{G} \nabla w \cdot \xi d x .
\end{aligned}
$$

Using the first equation in (17) and the boundary conditions together with $\mathrm{H} 1$, this may be rewritten as

$$
\int_{G}\left\{\nabla w \cdot B_{2}^{-1} \nabla w-\left(p_{2}+\alpha_{2} \cdot B_{2}^{-1} \alpha_{2}\right) w^{2}\right\} d x=\int_{\Gamma^{*}} w^{2} g_{2} d S .
$$

Now $u(x) \neq 0$ in $G$ implies (10) holds so that

$$
\int_{G}\left(\nabla w \cdot B_{1}^{-1} \nabla w-p_{1} w^{2}\right) d x \geqq \int_{\Gamma^{*}} w^{2} g_{1} d S .
$$

Combining this with (19) we arrive at a contradiction to (18) unless we have equality. According to Corollary 1.1, this latter occurs if, and only if, $u$ and $w$ are related by (11). This proves the theorem.

We remark that this result contains as special cases the theorems of Clark and Swanson [2] and Kreith [5]. We also note that the coefficients in (16) and (17) may depend on $(u, \zeta)$ and $(w, \xi)$ respectively as well as on $x$ so that the systems are quasilinear. Kreith [6] has recently proved a comparison theorem for two such systems using a generalized Picone-type identity. The order relation assumed in H3 may be replaced by a somewhat weaker one, but we must then assume a corresponding change in the order $r$ of zeros of $w$ on $\Gamma_{0}$.

Another comparison theorem between two systems of type (1) will now be derived. To do this we shall first establish another inequality similar to (9) in which a different assumption is used in place of the requirement $(\alpha-\beta) \cdot \varphi \geqq 0$. To this end we let $\lambda$ be the $d$-vector $\nabla w-w B \varphi$ and denote by $\lambda^{*}$ the $(d+1)$-vector $\lambda^{*}=(\lambda, w)$. Let $\theta=B^{-1}(\beta-\alpha) / 2=\left(t_{1}, \ldots, t_{d}\right)$ and let $E$ be the $(d+1) \times(d+1)$ matrix

$$
E=\left(\begin{array}{ll}
I_{d} & \theta \\
\theta^{\prime} & g
\end{array}\right),
$$


where $g$ is some scalar valued function and $\theta^{\prime}$ is the transpose of $\theta$. Denote by $q\left(\psi^{*}\right)$ the quadratic form in $(d+1)$ variables $\psi^{*}=\left(f_{1}, \ldots, f_{d+1}\right)$,

$$
q\left(\psi^{*}\right)=\psi^{*} \cdot E \psi^{*},
$$

and by $t_{i}^{*}$ the cofactor of $t_{i}$ in $E$.

THEOREM 3. Let $\varphi$ be a solution of (3) in G. Suppose there exists $g \in C(G)$ such that

$$
g \operatorname{det} B^{-1} \geqq \sum_{1}^{d} t_{i} t_{i}^{*}
$$

Then for every $w \in \Omega$ for which $\lim _{n} \int_{G_{n}} g w^{2} d x=\int_{G} g w^{2} d x$ exists, we have

$$
\int_{\partial G} w^{2} \varphi \cdot \eta d S \leqq \int_{G}\left\{\nabla w \cdot B^{-1} \nabla w+w \nabla w \cdot B^{-1}(\beta-\alpha)+(g-p) w^{2}\right\} d x
$$

Proof. It is known that condition (21) is both necessary and sufficient for the quadratic form (20) to be positive semidefinite, cf. [7]. It follows that

$$
\begin{aligned}
0 & \leqq \int_{G_{n}} q\left(\lambda^{*}\right) d x \\
& =\int_{G_{n}}\left\{B^{-1}(\nabla w-w B \varphi) \cdot(\nabla w-w B \varphi)+w \nabla w \cdot B^{-1}(\beta-\alpha)-w^{2}(\beta-\alpha) \cdot \varphi+g w^{2}\right\} d x \\
& =M\left(w: G_{n}\right)+\int_{G_{n}}\left\{w \nabla w \cdot B^{-1}(\beta-\alpha)-w^{2}(\beta-\alpha) \cdot \varphi+g w^{2}\right\} d x .
\end{aligned}
$$

Using ( $\left.8^{\prime}\right)$ and taking the limit as $n$ tends to infinity, we arrive at (22).

We are now ready to state a comparison theorem between the two first order systems

$$
\begin{aligned}
& \nabla u=u \alpha_{1}+B_{1} \zeta, \quad x \in G ; \quad u=0, \quad x \in \Gamma_{0}, \\
& \nabla \cdot \zeta=-p_{1} u+\beta_{1} \cdot \zeta, \quad x \in G ; \quad \zeta \cdot \eta=g_{1} u, \quad x \in \Gamma^{*} \text {, }
\end{aligned}
$$

and

$$
\begin{aligned}
& \nabla w=w \alpha_{2}+B_{2} \xi, \quad x \in G ; \quad w=0, \quad x \in \Gamma_{0}, \\
& \nabla \cdot \xi=-p_{2} w+\beta_{2} \cdot \xi, \quad x \in G ; \quad \xi \cdot \eta=g_{2} w, \quad x \in \Gamma^{*} \text {. }
\end{aligned}
$$

In addition to $\mathrm{H} 1, \mathrm{H} 2$ and $\mathrm{H} 3$ we also assume that $\beta_{i}$ satisfies the same hypotheses as $\alpha_{i}$. Moreover, we suppose the existence of a $g \in C(G)$ satisfying the same assumptions as $p_{i}$ and that (21) holds. Denote by

$$
\begin{aligned}
V(w)= & \int_{G}\left\{\nabla w \cdot\left(B_{1}^{-1}-B_{2}^{-1}\right) \nabla w+\left(g+p_{2}+\beta_{2} \cdot B_{2}^{-1} \alpha_{2}-p_{1}\right) w^{2}\right\} d x \\
& +\int_{G} w \nabla w \cdot\left[B_{1}^{-1}\left(\beta_{1}-\alpha_{1}\right)-B_{2}^{-1}\left(\beta_{2}-\alpha_{2}\right)\right] d x .
\end{aligned}
$$


THEOREM 4. Under the assumptions stated above, let $(w, \xi)$ be a solution of (24) such that $w \neq 0$ in $G$ and that $w$ has a zero of order $r>\frac{1}{2}$ on $\Gamma_{0}$. If $(u, \zeta)$ is a solution of (23) and if

$$
V(w)<\int_{\Gamma^{*}} w^{2}\left(g_{1}-g_{2}\right) d S,
$$

then $u$ must have a zero in $G$.

Except for the obvious changes the proof is entirely similar to that of Theorem 2 and will therefore be omitted.

We remark that Theorem 4 includes in particular a result of Swanson [7] on nonselfadjoint second order elliptic equations. The technique used here can also be applied to a single elliptic equation of the fourth order [9] as well as to matrix systems of second order elliptic equations [8].

ACKNOWLEDGement. This work was done while the author was visiting the Mathematical Institute, Tôhoku University, Sendai, Japan. The author wishes to thank Professors Taro Yoshizawa and Junji Kato and members of the Institute for their warm hospitality and many helpful conversations.

\section{REFERENCES}

1. P. R. Beesack, Integral inequalities of the Wirtinger type, Duke Math. J. 25 (1958), 477-498. MR 20 \#3947.

2. C. Clark and C. A. Swanson, Comparison theorems for elliptic differential equations, Proc. Amer. Math. Soc. 16 (1965), 886-890.

3. G. H. Hardy, J. E. Littlewood and G. Pólya, Inequalities, 2nd ed., Cambridge Univ. Press, New York, 1952. MR 13, 727.

4. P. Hartman, Ordinary differential equations, Wiley, New York, 1964. MR 30 \#1270.

5. K. Kreith, A strong comparison theorem for selfadjoint elliptic equations, Proc. Amer. Math. Soc. 19 (1968), 989-990.

6. —_, A Picone identity for first order differential systems, J. Math. Anal. Appl. 31 (1970), 297-308.

7. C. A. Swanson, A comparison theorem for elliptic differential equations, Proc. Amer. Math. Soc. 17 (1966), 611-616. MR 34 \#1663.

8. Pui-Kei Wong, Wirtinger type inequalities and elliptic differential inequalities, Tôhoku Math. J. 23 (1971), 117-127.

9. - Integral inequalities of Wirtinger-type and fourth order elliptic differential inequalities, Pacific J. Math. (to appear).

TôHoku University, Sendai, Japan

Current address: Michigan State University, East Lansing, Michigan 48823 